\title{
From Patient Data to Medical Knowledge: The Principles and Practice of Health Informatics
}

\section{Paul Taylor (2006). From Patient Data to Medical} Knowledge: The Principles and Practice of Health Informatics. Oxford: Blackwell Publishing Ltd. ISBN: 0-7279-1775-7

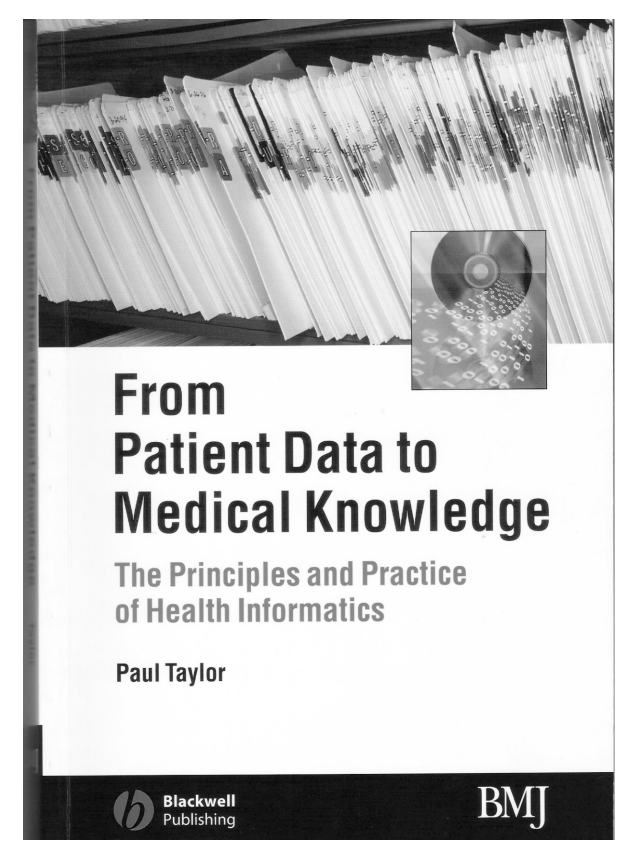

From Patient Data to Medical Knowledge: The Principles and Practice in Health Informatics is aimed at those who want to learn about how information technology is transforming the way we think about medicine and medical research.

The author, Dr. Paul Taylor, works in Centre for Health Informatics and Multiprofessional Education (CHIME) at the University College London (UCL). He is the director of the UCL postgraduate program in health informatics and has been running the program since 1999. He wrote this book on the basis of his long experience in teaching part-time students that mostly work in information or clinical roles in the National Health Service (NHS) or other health care organizations.

The prospective audience of this book is anyone who wants to learn to use information in health care: health professionals, hospital managers or IT specialists, etc. This book can be used as a textbook for a postgraduate course in health informatics.

The contents of this book consist of three parts.

Part 1 provides a broad introduction to the field of health informatics:

- Reading and writing patient records.

- Creation of medical knowledge.

- Access to medical knowledge.

Part 2 deals with various techniques used in health informatics and the theory behind some of these techniques. This involves a brief introduction to the essential techniques used to represent clinical concepts in computer programs such as electronic health care records or decision support systems and obtain any value out of the representation:

- Logic

- Controlled clinical terminology

- Knowledge representation

- Ontology.

- Clinical standards

- Probability and decision-making

- Probability and learning from data

Part 3 explores attempts to apply health informatics in practices. This includes implementation in clinical setting:

- Theories of organizational change 
From Patient Data to Medical Knowledge: The Principles and Practice of Health Informatics

- Attempts to change clinical practice by improving the dissemination of information

- Change management issues raised by attempts to introduce new technology into health care organizations

The greatest strength of this book lies in the practical examples which are drawn from health care organizations. While the overall content of this book is practical, a fundamental knowledge of information tech- nology is required to understand the germane material completely.

Hiye-Ja Lee, Ph.D. Yongin Songdam College, Dept. of Medical Information System hjlee@ysc.ac.kr 